\title{
Experimental treatment of ovarian cancers by adenovirus vectors combining receptor targeting and selective expression of tumor necrosis factor
}

\author{
SELVA R. MURUGESAN ${ }^{1}$, MASAKI AKIYAMA ${ }^{2}$, DAVID A. EINFELD ${ }^{1}$, \\ THOMAS J. WICKHAM ${ }^{1,3}$ and C. RICHTER KING ${ }^{1}$ \\ ${ }^{1}$ GenVec Inc, 65 West Watkins Mill Rd, Gaithersburg, MD 20878, USA; \\ ${ }^{2}$ FUSO Pharmaceutical Industries, Ltd., 2-3-30 Morinomiya, Joto-ku, Osaka, 536-8523, Japan
}

Received May 8, 2007; Accepted June 25, 2007

\begin{abstract}
Ovarian cancer is the fourth most common cancer among women and existing treatment is not routinely curative. One new strategy for cancer therapy is the selective delivery of $\mathrm{TNF} \alpha$ to tumors via adenovirus vectors. We have tested the combination of two modifications to adenovirus vectors designed to limit delivery to tumors, capsid modification and expression control. To target $\alpha_{\mathrm{v}} \beta_{3 / 5}$ integrin receptors that are highly expressed in tumor and sparsely expressed in the epithelial layer of peritoneum, we modified the capsid fiber and penton base to remove native receptor binding and incorporated an RGD-4C motif in the fiber knob (Ad.PB*F*RGD). This vector exhibits effective gene transfer in all of the $\alpha_{v} \beta_{3 / 5^{-}}$ positive ovarian cancer cells tested in vitro and in vivo. Importantly, the Ad.PB*F*RGD vector is able to transduce ovarian tumor nodules and avoid infecting the normal mesothelial cells that line the intraperitoneal space following intraperitoneal administration. To further increase selectivity, different promoters were incorporated into the capsid-modified vector to confer the expression of the hTNF $\alpha$ therapeutic gene. We analyzed both constitutive (CMV or RSV) and potentially tumor selective promoters (MUC-1, E2F or hTERT) in terms of efficacy, selectivity and safety. TNF-expressing $\mathrm{Ad} . \mathrm{PB} * \mathrm{~F} * \mathrm{RGD}$ vectors containing the MUC-1 promoter showed anti-tumor activity in two ovarian cancer xenograft models (Caov3 and Igr-ov1) with little evidence of toxicity or systemic TNF. The data indicate that combination of capsid modification and transcriptional regulation of expression is a
\end{abstract}

Correspondence to: Dr C. Richter King, GenVec Inc., 65 West Watkins Mill Rd, Gaithersburg, MD 20878, USA

E-mail: rking@genvec.com

Present address: ${ }^{3}$ EMD Lexigen, Bedford campus, 45A Middlesex Turnpike, Billerica, MA 01821, USA

Key words: adenovirus vector, tumor necrosis factor, gene therapy, RGD-4C, MUC-1/DF3, human ovarian cancer promising strategy for development of a new ovarian cancer treatment.

\section{Introduction}

There is a compelling need for new approaches to the treatment of ovarian cancer. It is estimated that 22,430 new cases of ovarian cancer will be diagnosed and 15,280 deaths from this cancer will occur in 2007 in the United States of America (1). There have been many improvements in the treatment of newly diagnosed ovarian cancer, however, most patients still recur and die of the disease. Improvement of the treatment of ovarian cancer will require efficacy against a disease that is disseminated within the peritoneal cavity. Metastatic spread outside the peritoneum is uncommon. Treatment of ovarian cancer involves surgical removal of intraperitoneal tumor masses (2) and chemotherapy to diminish disseminated cancer. Complete elimination of detectable tumor occurs in $\sim 60 \%$ of patients (3) and five-year survival rates of approximately $50 \%$ have been reported (4). Unfortunately, these treatments do not routinely remove all occult disease as relapse from ovarian cancer is very common, $>75 \%$, and primary disease failure nearly always occurs within the peritoneal cavity (5). Treatment of relapsed disease can involve surgery and chemotherapy. Chemotherapy for both primary and relapsed disease has been delivered either systemically or into the peritoneal cavity. Experimental therapies for ovarian cancer have the common goal of decreasing disease burden in the peritoneal space and include the addition of new cytotoxic agents, immune stimulation agents, and antibody based treatments (6). The confinement of ovarian cancer to the peritoneal space suggests that adenovirus vectors could provide a new treatment approach $(7,8)$. Delivery to the peritoneal space places the vector at high concentrations in close proximity to the target disease. As a result, approaches utilizing adenovirus vectors have undergone clinical testing as treatments for ovarian cancer $(5,9)$. The most advanced clinical studies involved vectors containing an expression cassette for the normal p53 gene $(5,10)$. Delivery of p53 protein to cancer cells having mutated p53 was expected to allow ovarian cancer cells to respond to apoptotic stimuli. Vectors that express a single chain antibody to erbB2 (11), a herpes virus TK gene $(12,13)$, the mda-7 gene 
(14) and a replication-selective vector (15) have also been clinically tested using intraperitoneal delivery. One important conclusion from previous clinical trials is that intraperitoneal delivery of adenovirus vectors was well tolerated (11). Doses of $>10^{13}$ particles per delivery were not associated with doselimiting toxicities (10). However, no clear anti-tumor activities were observed and improved potency may be required.

One approach to increase potency is the delivery of a secreted protein, TNF $\alpha$ to tumors. TNF is directly apoptotic and necrotic to tumor cells as well as causing destruction of tumor vasculature and activation of immune responses (16). Since systemic delivery of recombinant TNF protein results in significant dose limiting toxicities caused by vascular leakage (17), the only clinical application of recombinant TNF protein involves isolated limb perfusion for treatment of soft tissue sarcoma (18). The clinical activity of recombinant TNF $\alpha$ protein supports the concept of delivering a gene for TNF to tumors where it can be expressed and secreted causing the needed bystander effect without leading to significant systemic exposure to TNF. Such a strategy would require that expression be confined to the site of disease to limit systemic toxicities. A simple approach to confining TNF exposure is to directly inject into tumors an adenovirus vector containing the TNFa under a radiation inducible promoter $(19,20)$. Phase I testing of this agent indicates that direct injection into tumors was well tolerated with little evidence of systemic exposure to TNF. Partial or complete responses were seen in tumors derived from the breast, colon, pancreas, melanoma, rectum, lung and soft tissue sarcoma (21). In phase II testing in pancreatic cancer, at the maximum tolerated dose, approximately $60 \%$ of patients saw tumor shrinkage, improved survival, longer times of progression free survival and lower tumor markers. A further conclusion derived for preclinical and clinical testing of adenovirus vector expressing $\mathrm{TNF} \alpha$ is that anti-tumor responses are mediated by only a relatively small number of cells transduced in the tumor. Studies in animal models indicate that only $\leq 5 \%$ of cells contain an active gene following direct intra-tumoral injection (manuscript in preparation). These results suggest that the secreted nature of the TNF combined with TNF's breadth of biological activities might provide the increases in potency required for application in ovarian cancer. However, the systemic toxicity of TNF $\alpha$ is likely to require that expression must be confined to the site of disease.

Improvements in selectivity of adenovirus vectors can be accomplished by manipulating the capsid proteins that bind to cellular receptors and facilitate cell entry $(8,22)$. The primary receptor for adenovirus is the coxsackie adenovirus receptor (CAR) (23). CAR binding can be ablated through mutations of the fiber knob $(24,25)$. Secondary interactions occur through the penton base through binding to integrins (26) that can be ablated through removal of an RGD sequence in the penton (27). In order to target ovarian cancer cells we incorporated into the vector capsid a short peptide motif that binds a specific set of cell surface proteins, $\alpha_{v} \beta_{3 / 5}$ integrins, that are frequently expressed in ovarian and other cancers $(28,29)$.

A second approach to improving selectivity is to control gene expression through the choice of promoter $(7,8,30)$. Promoters can confer tumor selectivity according to tissue type (e.g. MUC-1), through cell cycle dependency (e.g. E2F), or by favoring immortalized cells (e.g. TERT). Expression of
MUC-1 is high in many adenocarcinomas including those derived from the breast, stomach, colon and ovary (31-33). Prevalence of expression of MUC-1 in ovarian cancers has been estimated as $>90 \%$ (34). E2F is elevated in ovarian (35) and brain (36) cancers. Likewise, hTERT is expressed in most human cancers (37) but inactive in normal somatic cells. The application of tumor selective promoters in adenovirus vectors has been used to generate replication-selective adenoviruses (38-40).

In the current study we explored the combination of capsid modification and promoter selectivity for delivery of $\mathrm{TNF} \alpha$ in an animal model of ovarian cancer. Our results indicate that such vectors can induce anti-tumor responses at doses where there is limited expression of TNF in normal tissue and no evidence of toxicity. We conclude that this combination of targeting strategies is a promising approach to the generation of new ovarian cancer treatments.

\section{Materials and methods}

Animals. Six week-old, immunocompetent female BALB/c or immunodeficient athymic nude, $\mathrm{nu} / \mathrm{nu}$, mice weighing 19-22 g were purchased from Harlan, USA, and were acclimated for 1 week, while caged in groups of five. The animals were housed in micro-isolator cages; each individually ventilated with HEPA supply and HEPA exhaust and maintained under conventional conditions. The animals were provided with a diet of regular global rodent chow (2018), purchased from Harlan, Teklad and sterilized or autoclaved water ad libitum throughout the experiment. The GenVec animal facility operates in accordance with the NIH Guide for the Care and Use of Laboratory Animals and complies with the PHS (Public Health Service) Policy on Humane Care and Use of Laboratory Animals. All of the cancer experimental procedures were conducted according to GLPs (Good Laboratory Practices) and were in compliance with the NIH guidelines and PHS policy for the care and welfare of animals.

Cell culture. Human ovarian cancer cells, A2780, Igr-ov1, Ovcar-4, Ovcar-5 and Ovcar-8 were obtained from NCI, Frederick, MD. The PA-1, ES2, Ovcar3 and Caov3 ovarian cancer cell lines were obtained from American Type Culture Collection, Manassas, VA. These cell lines were passaged 3-4 times before being used in experiments. A2780, Igr-ov1, Ovcar-3, Ovcar-4, Ovcar-5 and Ovcar-8 were cultured in RPMI-1640 (Cambrex, Walkersville, MD), whereas PA-1, ES2, and Caov3 were cultured in Dulbecco's modified Eagle's medium supplemented with $10 \%$ heat-inactivated FBS (HyClone, Logan, UT), 2 mM glutamine and antibiotics $(100 \mu \mathrm{g} / \mathrm{ml}$ of streptomycin, and $100 \mathrm{U} / \mathrm{ml}$ of penicillin). All the cultures were maintained at $37^{\circ} \mathrm{C}$ in a $5 \% \mathrm{CO}_{2}$ atmosphere.

Construction of recombinant adenovirus vectors. All the vectors described in this study are based on Ad5 with deletions in the E1, E3 and E4 regions. The deletion in E3 comprises the native $\mathrm{XbaI}$ fragment while the $\mathrm{E} 4$ region has been replaced with a transcriptionally inert spacer $(41,42)$. The AdL and AdGFP vectors express luciferase and GFP, using the CMV promoter and the SV40 early polyA sequence, with the expression cassette replacing nucleotides 356-3327 of the E1 
region and oriented with transcription directed toward the left end of the vector genome. The CMV promoter consists of nucleotides (-)583 to (+)167 relative to the transcription start site (Accession number X03922) and has a downstream splice acceptor prior to the transgene. The TNF vectors carry the gene for human TNFa (42) and differ from AdL and AdGFP in having an E1 deletion that extends from 356-3510 of Ad5.

Alternative promoters were inserted upstream of TNF by replacing nucleotides (-)583 to (+)74 of the CMV promoter, using the native SpeI and EagI sites at these positions in the E1-replacement shuttle plasmid. The human telomerase reverse transcriptase (TERT) and E2F promoters were generated by PCR amplification of genomic DNA from ARPE-19 cells (ATCC, CRL-2302). The primers ACTAGTCCCAGGACC GCGCTCCCCAC and CGGCCGGGGCCAGGGCTTC were used to amplify nucleotides (-)182 to $(+) 68$, relative to the transcription start site of TERT (AB016767) with flanking SpeI and EagI sites (added nucleotides underlined). Nucleotides (-)219 to (+)57 of the E2F promoter (AF516106) were amplified with flanking SpeI and NotI sites using the primers ACTAGTCTGGTACCATCCGACAAAGCCT and GCGGC CGCCTCGGCGAGGGCTCGATCC. PCR amplification of the MUC-1 promoter sequence flanked by SpeI and XhoI sites has been described previously (39). The SpeI-XhoI(blunt) fragment, carrying nucleotides (-)690 to (+)38 of the MUC-1 promoter (AL713999) was isolated and ligated into the SpeIEagI(blunt) shuttle plasmid. The SpeI-EagI(blunt) shuttle was also used for insertion of the RSV promoter as an SpeI-EcoRV fragment. The RSV fragment consists of nucleotides (-)340 to $(+) 34$ relative to the transcription start site of the promoter (L291299) followed by a 63-base spacer derived from the RSV expression plasmid.

The AdL.PB*F*HA vector has the penton base sequence HAIRGDTF replaced with TSYPYPDVPDYASS and an insertion of SRGFKSGSYPYDVPDYA between Gly ${ }^{543}$ and $\mathrm{Asp}^{544}$ of the fiber protein (HI loop) as described previously (43). In addition, the fiber knob has the mutations S408E, A415G, E416G and K417G to disable CAR binding. Vectors carrying an RGD motif in fiber have an insertion of CDCR GDCFC between Gly ${ }^{543}$ and Asp ${ }^{544}$ in the HI loop of the fiber knob together with the above CAR binding mutations. The GFP, luciferase, and TNF expressing RGD vectors do not have an HA epitope in the penton base, but only the SpeI linker that replaces HAIRGDTF with TS.

All vectors were expanded using 293-orf6 cells as previously described (43). Vectors were purified using 3 successive $\mathrm{CsCl}$ gradient centrifugation runs and dialyzed into formulation buffer. Purity and activity were monitored using standard methods (21). All preparations were active showing pu/FFU ratios $<32$.

In vitro gene transfer assays. Established human ovarian cancers such as PA-1, Ovcar-5, Caov3, Igr-ov1 and A2780 cells were cultured in 24 -well plates $\left(1 \times 10^{5}\right.$ cells/well) at $37^{\circ} \mathrm{C}$. These cell lines were infected with AdL (native tropism), AdL.PB*F* (CAR and integrin binding ablated) or AdL.PB*F*RGD (CAR and integrin binding ablated plus RGD-4C motif inserted in the fiber) vectors at $1 \times 10^{8} \mathrm{pu} /$ well for $1 \mathrm{~h}$. Following $1 \mathrm{~h}$ incubation at $37^{\circ} \mathrm{C}$, unbound virus in each well was removed, washed and replaced with
$1 \mathrm{ml}$ complete medium (DMEM or RPMI, 10\% FBS, $10 \mathrm{mM}$ HEPES). After the cells were incubated overnight in the incubator at $37^{\circ} \mathrm{C}, 5 \% \mathrm{CO}_{2}$, the cells were lysed with cell culture lysis buffer (Promega, Madison, WI) and the lysate was analysed using a luciferase assay system (Promega Corp, Madison, WI). The luciferase activity was measured in a TR717 microplate luminometer (Applied Biosystems, Bedford, MA) and expressed as RLU (relative light units)/20 $\mu 1$ lysate. All of the experiments were carried out in triplicate.

In vivo fluorescence microscopy. Human ovarian cancer Caov3 or Igr-ov1 cells $\left(1 \times 10^{7}\right.$ in $500 \mu 1$ serum free medium) were injected into the intraperitoneal cavity of athymic nude mice. After the tumor cells formed well-defined nodules on the surface of the various organs in the abdominal cavity, the vector administration was initiated. The GFP gene (controlled by CMV promoter) containing AdGFP $\left(1 \times 10^{11} \mathrm{pu}\right.$, GFP marker), and AdGFP.PB*F*RGD $\left(1 \times 10^{11} \mathrm{pu}\right)$ were injected into the intraperitoneal space of tumor bearing mice. Following 48 -h vector injection, mice were sacrificed. The abdominal cavity of each mouse was carefully opened and examined for tumor nodules. Then, the GFP expression in tumor, kidney, liver, lung, spleen, peritoneal membrane, mesentery and diaphragm was detected using a Leica (MZFLIII) fluorescence microscope with a separate filter for GFP. The images of GFP activity in each group were documented by a Spot RT digital camera (Diagnostic Instruments, Inc.).

In vivo measurement of luciferase marker expression. For the in vivo luciferase expression study, $2 \times 10^{10} \mathrm{pu}$ of either AdL or AdL.PB*F*RGD in $500 \mu 1$ volume was administered to the peritoneal cavity of Caov3 tumor bearing mice. One day following administration the animals were sacrificed and the tumor nodules, liver, spleen, kidneys, peritoneum and lungs were snap-frozen in liquid nitrogen. The frozen tissue was ground and a portion was lysed in cell culture lysis reagent (Promega). Luciferase activity in the supernatant was determined as described above. Protein concentrations determined by Bradford protein assay (BioRad) were used to normalize the luciferase activities.

In vitro quantification of TNF $\alpha$. Human ovarian carcinoma (PA-1, Caov3, and Igr-ov1) cell lines were seeded in 48-well plates $\left(1 \times 10^{5}\right.$ cells/well $)$. The cells were infected with vectors, AdCMV.TNF.PB*F*RGD, AdRSV.TNF.PB*F*RGD, AdE2F.TNF.PB*F*RGD, AdMUC-1.TNF.PB*F*RGD and AdhTERT.TNF.PB*F*RGD at $1 \times 10^{9} \mathrm{pu} /$ well for $24 \mathrm{~h}$ at $37^{\circ} \mathrm{C}$, $5 \% \mathrm{CO}_{2}$. The control wells received medium only. Then, the cells were lyzed using cell culture lysis reagent (1x) (Promega) for $30 \mathrm{~min}$ at room temperature followed by a freeze-thaw cycle. The hTNF $\alpha$ concentrations were determined using a hTNF $\alpha$ ELISA kit (Pierce, Rockford, IL) with a level of detection of $<2 \mathrm{pg} / \mathrm{ml}$.

Development and treatment of peritoneal tumor model of human ovarian cancer. Tumor cells, Caov3 or Igr-ov1, $\left(1 \times 10^{7}\right.$ cells $)$ in a volume of $500 \mu 1$ serum-free medium containing $10 \mathrm{mM}$ HEPES were introduced into the peritoneal cavity of female athymic nude mice (8-10 weeks). Three mice were sacrificed 4 or 5 days after cell inoculation and a 


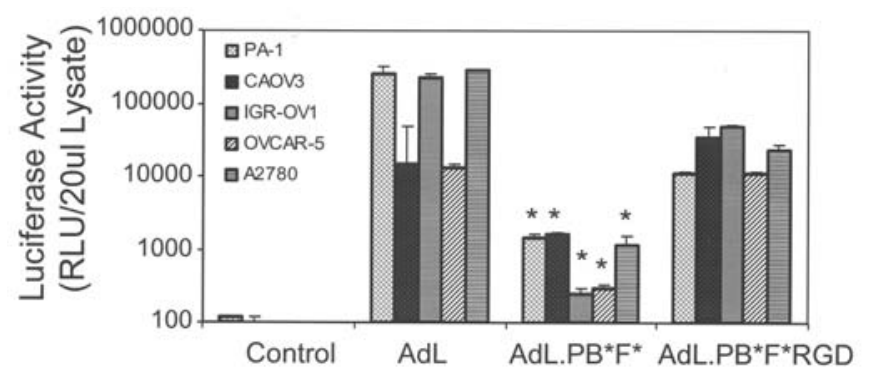

Figure 1. Comparison of in vitro gene delivery profiles of untargeted and targeted vectors in established human ovarian cancer cell lines. The ovarian cancer cells, PA-1, Caov3, Igr-ov1, Ovcar-5 and A2780 were cultured in 24-well plates $\left(1 \times 10^{5}\right.$ cells/well) and infected with vectors at $1 \times 10^{8} \mathrm{pu} /$ well for $1 \mathrm{~h}$. Luciferase activity was measured and reported as relative light units (RLU) per $20 \mu 1$ lysate. Each bar represents the mean value of triplicates and the error bars represent standard deviation. The statistical analysis of the data was performed by two sample independent t-test. ${ }^{*}$ Statistically significant difference from the AdL or AdL.PB*F*RGD ( $<<0.05)$. The RGD-4C adenovirus vector is capable of delivering genes more efficiently to a variety of human ovarian cancer cell lines relative to ablated vectors. The AdL vector possesses intact native tropism. The AdL.PB*F* vector contains no CAR and integrin binding. The AdL.PB*F*RGD vector contains no CAR and integrin binding but possesses the RGD-4C motif in the HI loop of the fiber.

thorough evaluation of all of the organs and tissues in the abdominal cavity was performed by gross examination. The mice showed 2-mm nodules (Caov3) or 2- to 5-mm (Igr-ov1) distributed on the surface of the omentum, peritoneum, liver, pancreas, diaphragm and mesentery. No mice developed ascites at this stage. Additionally, no tumor formation was evident in the pleural cavity. A time point of 4 or 5 days post tumor cell inoculation was selected to initiate treatment for efficacy experiments. We tested these two xenograft models (Caov3 and Igr-ov1) in athymic nude mice. The vector in $\sim 500 \mu$ l final formulation buffer was administered into the ip cavity of nude mice. Each mouse received vector treatment three times over a period of seven days (on 4, 7, and 11 days post tumor cell inoculation). The volume of the vector solution for each mouse was calculated on the basis of $20 \mathrm{~g}$ body weight. Thirty or thirty-five days after treatment initiation, mice were sacrificed. The necropsy analysis was blinded and performed carefully by gross examination. All visible tumors were collected and weighed.

Determination of TNF a gene expression after intraperitoneal application. Naïve BALB/c mice were segregated into four different groups, control [FFB (final formulation buffer)], AdCMV.TNF.PB*F*RGD, AdRSV.TNF.PB*F*RGD and AdMUC-1.TNF.PB*F*RGD. Each experimental group contained 4 mice. Each mouse received a single intraperitoneal injection of the respective vector at a dose level equivalent to the tolerable dose in $500 \mu \mathrm{l} \mathrm{FFB}$. Blood and peritoneal lavage were collected from each mouse on days 1, 4 and 7 following vector administrations. Peritoneal fluid was collected by injection of $1.5 \mathrm{ml}$ PBS, followed by gentle massage of the abdomen for $1 \mathrm{~min}$. The peritoneal lavage $(1 \mathrm{ml})$ was collected, centrifuged and stored at $-80^{\circ} \mathrm{C}$. Blood was collected in an eppendorf tube directly from the heart by cardiac puncture. Blood was kept on ice immediately for $1 \mathrm{~h}$ after the collection and then it was centrifuged at $6700 \mathrm{rpm}$ at $4^{\circ} \mathrm{C}$ for $14 \mathrm{~min}$. The serum was separated and stored at $-80^{\circ} \mathrm{C}$ until further analysis. The hTNF $\alpha$ concentrations in the serum and peritoneal lavage were measured by ELISA using a Quantikine hTNF $\alpha$ enzyme-linked immunosorbent assay kit (R\&D Systems, Minneapolis, MN) with a level of detection of $<4.4 \mathrm{pg} / \mathrm{ml}$.

Statistics. Data are presented as mean values \pm standard deviation. Statistical analyses were performed with Origin 7.5 software (Origin Laboratories, Northampton, MA). A Shapiro-Wilk normality test was performed to determine whether the samples followed a normal distribution. For normally distributed data, the means of treatment groups were compared by one-way ANOVA for more than two groups. Tukey multiple comparisons were used to specify which groups differ at the predetermined $p<0.05$. Twosample independent t-test was also employed to compare means between two groups. If the data were not normally distributed, the Wilcoxen signed-rank test was performed. In all cases, p-values of $<0.05$ were considered significant.

\section{Results}

Targeting adenovirus vectors using $R G D-4 C$. Adenovirus vectors containing mutations within the fiber knob and penton base to prevent binding to native receptors have been previously reported (43). Using such doubly ablated vectors as a base, we introduced the peptide CDCRGDCFC (RGD-4C) into the HI loop of the fiber knob to alter the primary receptor specificity to $\alpha_{v} \beta_{3 / 5}$ integrins. To determine if the RGD-4C ligand permits vector transduction of established human ovarian cancer cells, we tested a panel of cell lines (PA-1, Caov3, Igr-ov1, A2780 and Ovcar-5). Fig. 1 demonstrates that the AdL vectors, possessing wild-type receptor binding, easily transduce all cell lines. Doubly ablated vectors (AdL.PB*F*) lacking native receptor binding have significantly less ability to transduce these cells as measured by luciferase activity. This vector was shown to be fully functional for entry into cells through testing on a cell line containing an artificial receptor capable of binding the fiber knob. Addition of the RGD-4C motif into the doubly ablated vector (AdL.PB*F*RGD) restored the ability significantly $(\mathrm{p}<0.05)$ to transduce ovarian cancer cell lines (Fig. 1).

Intraperitoneal models of human ovarian cancer. Human ovarian cancer forms extensive solid tumor carcinomatosis in the peritoneal space. To model this disease we examined the timing and morphology of tumor growth by human cell lines in nude mice. PA-1, Ovcar3, Caov3, SW626, A2780, Igr-ov1, Ovcar4, Ovcar-5 and Ovcar8, were assessed in nude mice on day 4,8 and 15 after tumor cell inoculation $\left(1 \times 10^{7}\right.$ cells/ $500 \mu \mathrm{l})$. Solid tumor nodules were found on the surface of the peritoneum, intestine, liver, diaphragm, pancreas, omentum and mesentery in each mouse (data not shown). Ascites was not found in any animals during the observation period. The PA-1, Caov3 and Igr-ov1 developed many fine nodules $(\sim 2 \mathrm{~mm})$ between 4 and 8 days after tumor cell inoculation. The cell lines Igr-ov1 and Ovcar-5 grew very quickly with 4- to 12 -mm tumor nodules at 15 days post implant. With all the tumor cell lines, no metastases were detected in organs outside the peritoneal cavity. Based on this observation we 


\section{Tumor Nodules}
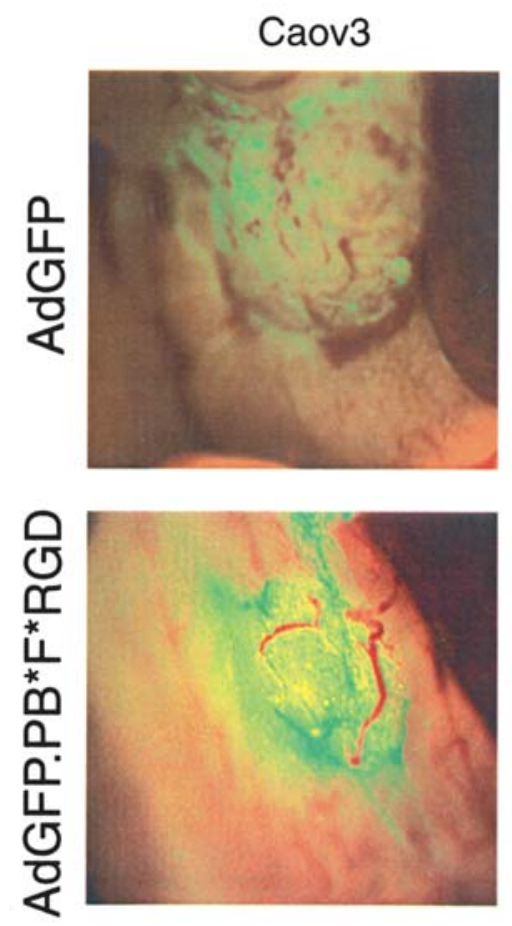
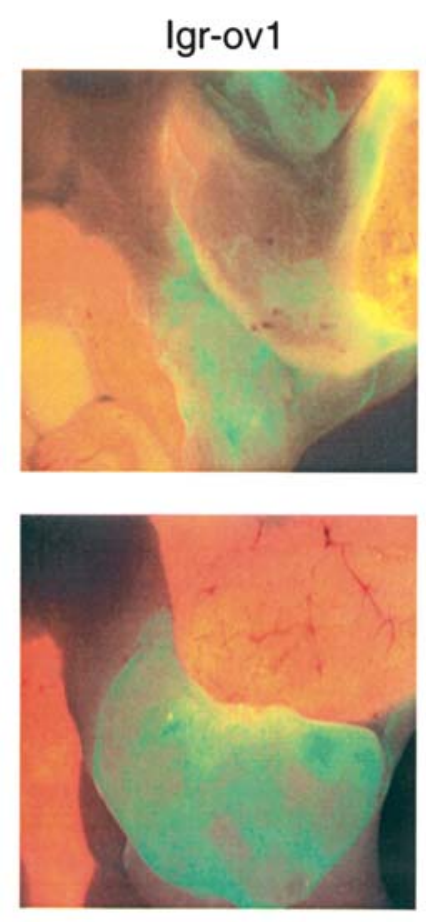

\section{Normal Tissues}
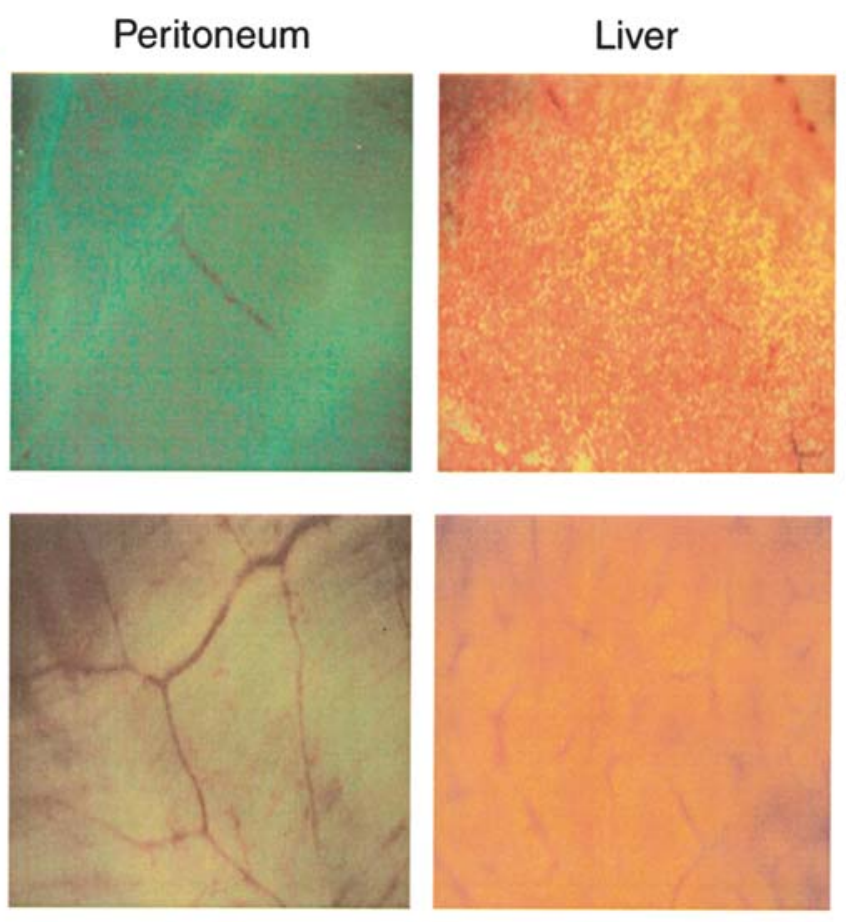

Figure 2. Comparison of in vivo gene delivery profiles of untargeted and targeted vectors in human ovarian tumor growing mice. Human ovarian tumor nodules (Caov3 or Igr-ov1) were developed in the intraperitoneal (ip) space of athymic nude mice. The green fluorescence protein (GFP) activity was observed $48 \mathrm{~h}$ following vector $\left(1 \times 10^{11} \mathrm{pu} / 500 \mu \mathrm{l}\right)$ administration into the ip space of tumor bearing mice (n=3). Photomicrographs were taken of whole tissue exposed on dissection of the ip cavity. The peritoneum and liver micrographs are from Igr-ov1 tumor bearing mice. The capsid modified virus with the HI loop RGD-4C motif in the fiber promotes gene transfer to Igr-ov1 or Caov3 tumor nodules located in the ip cavity while avoiding mesothelial cells that line the peritoneal space and major organs.

selected one slower growing tumor line Caov3 and one fast growing line Igr-ov1 for further testing. These results indicated that established tumor nodules of approximately $2 \mathrm{~mm}$ should be present at 4 or 5 days post tumor cell inoculation.

Selective gene delivery by $R G D-4 C$ Ad vectors in the peritoneal cavity. Previously, we demonstrated that the capsid modified vectors, via ablation of both CAR and integrin binding, dramatically reduced gene transfer to normal tissues after IP injection (44). Here, we tested the effect of adenovirus capsid modifications on gene transfer to ovarian tumor nodules and normal tissues in vivo. Vectors with wild-type tropism (AdGFP) and vectors without native tropism but containing RGD-4C in the fiber knob (AdGFP.PB*F*RGD) were compared. All contained the same marker gene expression cassette in which the green fluorescent protein (GFP) coding sequence is placed under the direction of the CMV promoter. Tumor nodules were established in the ip space of nude mice by injecting Caov3 or Igr-ov1 cell lines. Following single ip injection of wildtype vector, AdGFP, expression was detected in the tumor nodules, peritoneal wall, liver (Fig. 2), mesentery, lung, kidney, spleen and diaphragm (data not shown). Importantly, the AdGFP.PB*F*RGD vector transduced tumor nodules but reduced GFP activity was detected in normal tissue. To determine the levels of reporter gene expression, vectors encoding the luciferase reporter gene were administered to Caov3 tumor-bearing mice and assayed for luciferase activity in the tumor and abdominal organs (Fig. 3). As above, a

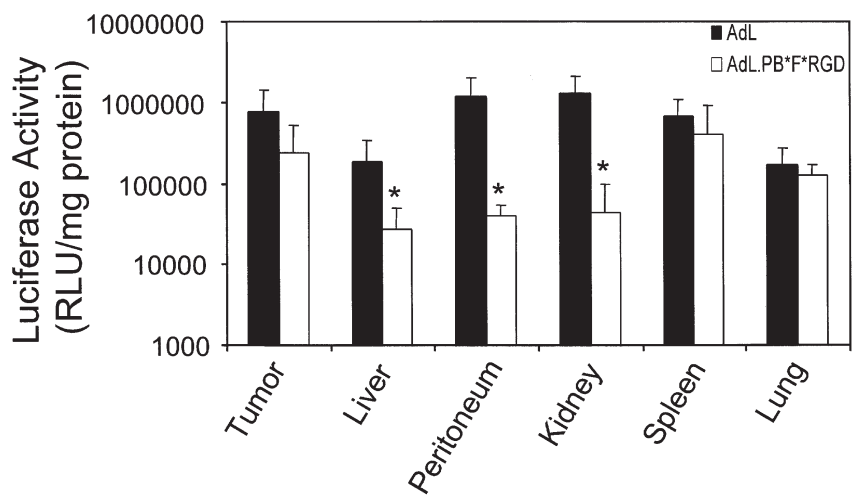

Figure 3. Luciferase reporter gene expression following intraperitoneal administration of targeted vector. Caov3 ovarian cancer cells were implanted into the peritoneal cavity of athymic nude mice. After the tumor nodules were established in the peritoneal cavity, indicated vectors were intraperitoneally administered at a dose of $2 \times 10^{10} \mathrm{pu}$. The next day the organs were resected for luciferase measurement. The means of luciferase activity in the target tumor and non-target organs were shown. Closed black column, AdL $(n=7)$; open column, AdL.PB*F*RGD $(n=9)$. Error bars represent standard deviation. Note that significant reduced transductions were observed in the liver, kidney and peritoneum ( $\mathrm{p}<0.05$, two sample independent t-test) from AdL.PB*F*RGD-treated animals relative to AdL-treated animals. * $\mathrm{p}<0.05$ compared with AdL.

vector with wild-type tropism (AdL) was compared with the vector that lacked native receptor binding but contained RGD-4C in the fiber knob (AdL.PB*F*RGD). The modified 
Table I. Comparison of CMV, RSV, E2F, MUC-1 and hTERT promoter activity in established human ovarian cancer cell lines.

\begin{tabular}{lccr}
\hline Promoters & CAOV3 & IGR-OV1 & PA-1 \\
\hline CMV $^{\text {a }}$ & $24000 \pm 2200$ & $25600 \pm 1000$ & $1100 \pm 30$ \\
RSV & $40 \pm 5$ & $500 \pm 80$ & $100 \pm 3$ \\
E2F & $60 \pm 1$ & $270 \pm 10$ & $80 \pm 4$ \\
MUC-1 & $80 \pm 10$ & $150 \pm 10$ & $0 \pm 0$ \\
hTERT & $9 \pm 7$ & $300 \pm 10$ & $10 \pm 1$ \\
\hline
\end{tabular}

The ovarian cancer cell lines, PA-1, Caov3, and Igr-ov1 were cultured in 48 -well plates $\left(1 \times 10^{5}\right.$ cells/well). The cells were infected with RGD targeted vectors with hTNF $\alpha$ under the control of the indicated promoters at $1 \times 10^{9} \mathrm{pu} /$ well. Following 24-h incubation, the hTNF $\alpha$ expression was determined. Results shown are mean values $(\mathrm{pg} / \mathrm{ml})$ of duplicate infections \pm standard deviation. All the promoters appear to be positive in all the ovarian cancer cell lines. Of note, CMV exhibited significantly higher activity than other promoters $(\mathrm{p}<0.05)$. PA-1 lacks MUC-1 promoter activity. ${ }^{\mathrm{a}} \mathrm{p}<0.05$ versus all other promoters (one-way ANOVA).

vector showed significantly reduced transductions of liver $(\mathrm{P}<0.05)$, kidney and peritoneum $(\mathrm{P}<0.005)$. Levels of gene expression were similar in the target tumor. When the transduction was normalized to the level of tumor transduction, the AdL.PB*F*RGD vector gave reductions relative to AdL of $57 \%, 78 \%$ and $78 \%$ for liver, kidney and peritoneum transduction respectively. These data indicated that the RGD$4 \mathrm{C}$ vector achieved selective gene delivery to tumor nodules in vivo by retaining the interaction with tumor cells while reducing the transduction to some normal abdominal organs.
As a consequence of these promising improvements in the selectivity for ip administration we focused all additional analysis on vectors containing both ablations in the native adenovirus receptor interactions and the RGD-4C targeting ligand.

$R G D-4 C$ Ad vectors that provide selective expression of $T N F \alpha$. We have previously reported that vectors containing deletions in the wild-type receptor interactions can exit the peritoneal cavity as evidenced by trafficking to the liver and other organs (44). Similarly, the RGD-4C targeted vector is detected in samples of spleen and lung following administration into the peritoneal space (Fig. 3). Consequently, systemic exposure to vectors cannot be ruled out. This indicates that other mechanisms to provide tumor selectivity may be desirable. We tested different promoters driving the expression of $\mathrm{TNF} \alpha$ as possible strategies to increase the selectivity of gene product delivery. We created vectors that contain the CMV, RSV, E2F, hTERT, and MUC-1 promoters configured to drive expression of $\mathrm{TNF} \alpha$. We selected these promoters as they have been associated with rapidly proliferating cells (E2F), immortalized cells (hTERT) and with upregulation in adenocarcinoma (MUC-1). Other elements of the genome of the adenoviral vector genome remain identical between the vectors including the $\mathrm{E} 3$ and $\mathrm{E} 4$ deletion.

We assessed the expression of $\mathrm{TNF} \alpha$ using established human ovarian cancer cell lines (PA-1, Caov3, and Igr-ov1). The results demonstrate that among the five promoters examined, the CMV promoter is the strongest for TNF $\alpha$ expression in all the ovarian cancer cells (Table I). The tumor selective promoters, MUC-1, hTERT and E2F induce lower expression comparable to the RSV promoter. For the PA-1 tumor cell line no TNF $\alpha$ expression was detectable following the infection of the MUC-1 driven vector. This is consistent with the reported adenocarcinoma selectivity of the MUC-1

Table II. Evaluation of the tolerable dose of RGD-targeted adenovirus vectors that drive TNF protein under the control of a viral or tumor-specific promoter.

\begin{tabular}{lcccc}
\hline Vectors & Dose & Mortality $(\%)$ & Body weight loss (\%) & Tolerable dose \\
\hline FFB & & 0 & 0 & NA \\
AdCMV.TNF.PB*F*RGD & $3.00 \mathrm{E}+10$ & 0 & $12.7 \pm 1.3$ & $3.00 \mathrm{E}+10$ \\
& $6.00 \mathrm{E}+10$ & 0 & 0 & $17.2 \pm 3.1$ \\
AdRSV.TNF.PB*F*RGD & $6.00 \mathrm{E}+10$ & 0 & $8.4 \pm 1.6$ & $1.20 \mathrm{E}+11$ \\
& $1.20 \mathrm{E}+11$ & 0 & 0 & 0 \\
AdE2F.TNF.PB*F*RGD & $6.00 \mathrm{E}+10$ & 0 & 0 & $1.20 \mathrm{E}+11$ \\
& $1.20 \mathrm{E}+11$ & 0 & 0 & $1.20 \mathrm{E}+11$ \\
AdMUC1.TNF.PB*F*RGD & $6.00 \mathrm{E}+10$ & 0 & 0 & 0 \\
& $1.20 \mathrm{E}+11$ & 0 & $3.6 \pm 2.3$ & $1.20 \mathrm{E}+11$ \\
AdhTERT.TNF.PB*F*RGD & $6.00 \mathrm{E}+10$ & 0 & 0 & 0 \\
\end{tabular}

Naïve Balb/c mice $(n=5)$ were given a single ip injection of vector at the indicated dose. Body weight loss at day two and mortality were noted. Body weight loss is listed as the mean percentage of loss \pm the standard deviation. Tolerable dose was selected as the highest dose that caused no mortality and $<20 \%$ weight loss. FFB, final formulation buffer; NA, not applicable. 


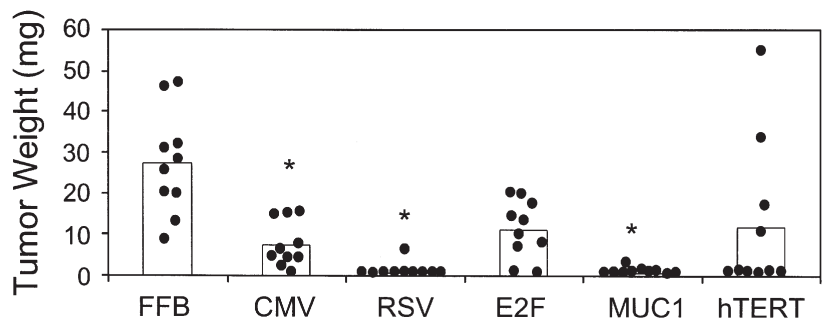

Figure 4. Tumor-specific promoter control of hTNF $\alpha$ expression promotes efficacy on human ovarian cancer cells, Caov3. Tumors were developed in the intraperitoneal cavity (ip) of athymic nude mice $(n=10)$. Mice received ip vector (CMV, RSV, E2F, MUC-1 and hTERT) administration at a tolerable dose three times each over a week. Control mice received final formulation buffer (FFB). Following 30-day treatment initiation, all mice ( $n=10 /$ group) were sacrificed. Tumors were removed and weighed. Each point represents the tumor weight from one mouse, and the bar represents the mean tumor weight of the group. As the data distribution was not normal in the hTERT group, statistical analysis was performed using the Wilcoxon rank sum test. Significant reduction of tumor burden was found in CMV, RSV and MUC-1 relative to FFB. Notably, six out of ten mice were tumor free following treatment with vector that contains hTNF $\alpha$ under the control of MUC-1. ${ }^{*} \mathrm{p}<0.05$ versus FFB.

promoter as PA-1 is derived from a teratocarcinoma showing no MUC-1 expression (39).

Efficacy of RGD-4C Ad vectors. To conduct efficacy testing, we first established a dose that could be safely administered, causing $<20 \%$ body weight loss and no evident morbidity (Table II). The maximum dose of CMV-driven vector was $3 \times 10^{10} \mathrm{pu} / 20 \mathrm{~g}$ body weight $(\mathrm{BW})$. All the other vectors caused no significant body weight loss up to $1.2 \times 10^{11} \mathrm{pu} / 20 \mathrm{~g} \mathrm{BW}$.

Efficacy testing was conducted in two intraperitoneal tumor models of human ovarian cancer (Caov3 and Igr-ov1) in athymic nude mice. We initiated treatment under conditions where established tumor nodules were known to be present.

We first screened all vector constructs for activity using the Caov3 cell line. Treatment was initiated five days after tumor cell inoculation, and a total of three injections were administered over seven days. The animals' behavior and body weights were monitored daily for any sign of morbidity. Thirty days following treatment and in a manner where the investigator was blinded to the treatment group, tumors were harvested and weighed. Fig. 4 illustrates the weight of tumor nodules following treatment with CMV, RSV, MUC-1, E2F and hTERT. Vectors containing the CMV, RSV and MUC-1 promoters showed significant reductions $(\mathrm{p}<0.05)$ in tumor burden compared to mice treated with control media. E2F and hTERT showed no statistically significant anti-tumor effect. The size of tumor nodules in animals that received vector with MUC-1 or RSV promoter was significantly reduced. Six out of 10 mice were tumor free when treated with vectors containing the MUC-1 promoter.

To confirm that the MUC-1 and RSV containing RGD-4C targeted vectors can show efficacy against a more aggressive ovarian cancer model, we tested the human cell line Igr-ov1. We compared vector treatment to FFB treatment. We determined that the Igr-ov1 cells rapidly form solid nodules of approximately $4 \mathrm{~mm}$ on the surface of the pancreas, liver, omentum, peritoneum, mesentery, ovary, and low abdominal fat within 4 days after tumor cell inoculation. We used a

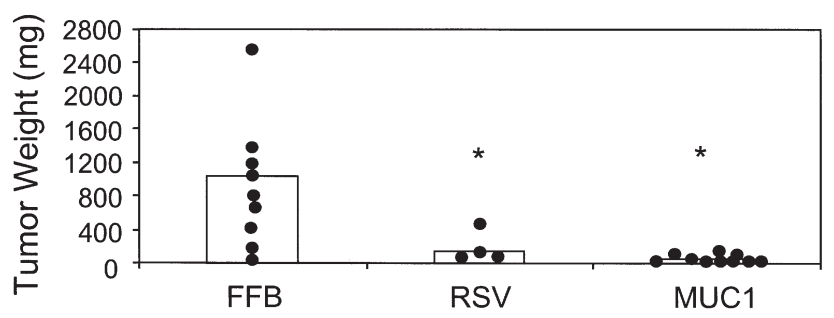

Figure 5. Tumor-specific promoter control of hTNF $\alpha$ expression is also efficacious on fast growing human ovarian cancer cells, Igr-ov1. Tumors were grown in the intraperitoneal (ip) cavity of athymic nude mice. The mice were treated with the MUC-1 $(n=10)$ or RSV $(n=5)$ vector at a tolerable dose three times each over a week. Control mice $(n=10)$ received final formulation buffer (FFB) alone. Following 30-day treatment initiation, all mice were sacrificed. Tumors were removed and weighed. Each point represents the tumor weight from one mouse, and the bar represents the mean tumor weight of the group. Note that one animal from the FFB and RSV group died. Significant anti-tumor activity was found in both RSV and MUC-1 vector-treated mice related to FFB-treated mice. ${ }^{*} \mathrm{p}<0.05$ versus FFB (one-way ANOVA followed by the Tukey multiple comparisons).

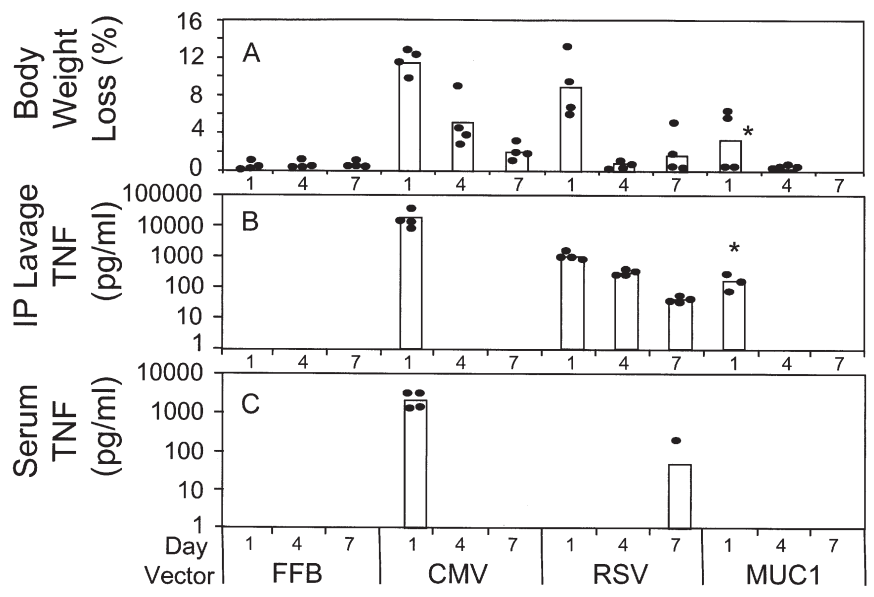

Figure 6. Tumor-specific promoter control of hTNF $\alpha$ expression promotes safety. The body weight (A) and hTNF $\alpha$ expression in intraperitoneal fluid (B) and serum $(C)$ of naïve BALB/c mice $(n=4)$ were measured following a single administration of CMV, RSV, or MUC-1 promoter-controlled hTNF $\alpha$ vectors at a tolerable dose. Control mice received $500 \mu 1$ of final formulation buffer (FFB) alone. Each point represents the value from one mouse, and the bar represents the mean value of the group $(n=4)$. One-way ANOVA analyses were performed. Reduced TNF level and body weight loss were found on day 1 in mice treated with RGD-4C TNF vector that contains MUC-1, relative to $\mathrm{CMV}$ or RSV promoter containing vectors. ${ }^{*} \mathrm{p}<0.05$ versus $\mathrm{CMV}$ or RSV.

similar dosing scheme as used above, three times $1.2 \times 10^{11} \mathrm{pu} /$ $20 \mathrm{~g}$ over one week. Both MUC-1 and RSV vectors displayed reduced tumor burden compared to control mice (Fig. 5).

$R G D-4 C$ Ad vectors can reduce systemic exposure to TNF $\alpha$. We measured the level of $\mathrm{TNF} \alpha$ protein in the serum and intraperitoneal fluid lavage of non-tumor bearing mice following a single ip injection at the tolerable dose (Fig. 6). Vectors containing the CMV and RSV promoters resulted in $\mathrm{TNF} \alpha$ in both serum and ip fluid. $\mathrm{TNF} \alpha$ induced by the CMV promoter was transient with a peak level at $24 \mathrm{~h}$ after administration and decreased to non-detectable by 4 days. Expression from the RSV promoter was lower than CMV at $24 \mathrm{~h}$, but unlike CMV, detectable expression persisted for at 
least 7 days. Low levels of TNF $\alpha$ were detected only at $24 \mathrm{~h}$ in ip lavage fluid following administration of vector containing the MUC-1 promoter. Using this vector $\mathrm{TNF} \alpha$ levels were not detected in serum at any time point. Body weights of the animals were monitored as a measurement of $\mathrm{TNF} \alpha$-associated toxicity. A significant drop in body weight of $>8 \%$ was noticed at day 1 after CMV, or RSV vector application $(\mathrm{p}<0.05)$. Importantly, body weight loss was $<3 \%$ with MUC-1. For CMV and RSV containing vectors, the observed elevations in serum or ip TNF levels correlate well with body weight loss. These results suggest that the MUC-1 promoter accounts for the reduced expression of TNF $\alpha$ in non-target tissues compared to the non-regulated viral promoters and that this may improve tolerability.

\section{Discussion}

Our results indicate that the improved design of adenovirus vectors for ovarian cancer treatment is possible. The results extend preclinical and clinical evidence that delivery of TNF $\alpha$ using adenovirus vectors may have anti-tumor activity. As a secreted molecule, TNF $\alpha$ can be expected to supply a strong bystander effect. We believe that this bystander effect of TNF $\alpha$ is necessary for the antitumor activity observed in this study of ovarian cancer. This view is supported by our results using targeted vectors containing the marker gene GFP, clearly indicating heterogeneity of transduction within tumors. The results in the present study support the conclusion that delivery of TNF $\alpha$ using adenovirus vectors causes only anti-tumor effects. There are reports that indicate that TNF $\alpha$ can play a tumor promotion role (45-47). However, we have not seen any evidence of increased tumor burden following delivery of any TNF expressing vector at any dose level.

In this study we extend our previous results and those of other groups showing that alteration in the virus capsid can produce improvements in selective transduction of target cells $(43,48,49)$. The RGD-4C ligand used in this study has been reported to bind to $\alpha_{v} \beta_{3}$ and $\alpha_{v} \beta_{5}$ integrins $(48,49)$. The frequency of $\alpha_{v} \beta_{3}$ and $\alpha_{v} \beta_{5}$ integrins in ovarian cancers has been estimated to be $62 \%$ and $65 \%$ respectively $(28,50)$. Our results showing efficient transduction of ovarian cancer cell lines by RGD-4C vectors are consistent with these findings. We have previously reported that receptor-ablated vectors show reduced transduction of non-target tissue of the peritoneal space (44). In the present study similar results were obtained with vectors having both native receptor ablation and containing the RGD-4C ligand in the fiber knob. The RGD-4C adenovectors show clear advantages for intraperitoneal delivery over adenovector with wild-type receptor binding. Of particular interest was the limited delivery of RGD-4C targeted vector to the peritoneal wall since intraperitoneal administration will clearly bring vectors in contact with the thin surface membrane composed of mesothelial cells. A study of human biopsy samples of the mesothelial membrane (51) shows that $\alpha_{v}$ subunits were not detected in the mesothelial layer that surrounds the peritoneal space. Some integrins ( $\alpha 2, \alpha 3$ and $\alpha 6)$ were expressed while others $\left(\alpha 1, \alpha 4, \alpha 5\right.$ and $\left.\alpha_{v}\right)$ were not observed (51). We interpret this study to indicate that our vectors containing the RGD-4C ligand may have limited targets on the normal lining of the peritoneal cavity in humans. We recognize that other studies of cultured peritoneal mesothelial cells and ascites-derived cells show $\alpha_{v} \beta 3$ and $\alpha_{v} \beta 5$ expression $(29,52)$. This has led others to predict that the RGD-4C adenovirus vectors will have limited application in the peritoneal cavity.

Our most encouraging results were obtained using the MUC-1 (DF3) promoter. The MUC-1 promoter may have particular advantages for ovarian cancer application as it can be upregulated in this disease $(33,34)$. Tai et al $(33)$ also studied a replication-deficient AdDF3-Bgal vector in which the B-gal gene was placed under the control of the MUC-1 promoter and showed selective B-gal expression in the ip tumor and no non-specific B-gal expression in the mesothelial cells of the peritoneum following ip administration. A similar finding was also demonstrated using non-replicating adenovirus with the MUC-1 promoter and transduction of MCF-7 cells (53). Our results show limited efficacy when TNF $\alpha$ expression was placed under direction of the E2F or telomerase promoters. For other applications and delivery methods these promoters may have important advantages. Other investigators have demonstrated tumor-selective gene expression using cell-type or tissue-type specific promoters, DF3/MUC-1 (33), E2F (36), hTERT (37), Cox-2 $(38,40)$ and SLPI $(54)$.

Our results convince us that the combination of native receptor binding ablation, RGD-4C targeting, and tumor selective expression of $\mathrm{TNF} \alpha$ provides a means to overcome many of the limitations of the adenovirus vectors previously tested in clinical trials for ovarian cancer. Ongoing studies in our laboratory are examining the relative contribution of capsid targeting and expression control to allow selective delivery of TNF to tumors in the intraperitoneal space. Other investigators are also exploring strategies to improve the activity and selectivity of adenovirus vectors for ovarian cancer application. Preclinical investigations have been initiated with RGD containing replication-defective and replication-selective adenoviruses $(38,52,54-56)$. It is hoped that a new generation of highly selective adenovirus vectors can provide better treatment for patients with ovarian cancer.

\section{Acknowledgements}

We acknowledge the expertise of Dawn Hritz in cell culture and in vitro studies, Randy Osborn and Leslie West for animal studies and Kenji Kawagishi for in vivo luciferase biodistribution study. Grant support: National Cancer Institute SBIR \# 1R43CA107658-01 (C. Richter King).

\section{References}

1. American Cancer Society: Cancer Facts and Figures. American Cancer Society, Atlanta, GA, 2007.

2. McCreath WA and Chi DS: Surgical cytoreduction in ovarian cancer. Oncology 18: 645-653, 2004.

3. Bookman MA, McGuire WP, Kilpatrick D, et al: Carboplatin and paclitaxel in ovarian carcinoma: a phase I study of the Gynecologic Oncology Group. J Clin Oncol 14: 1895-1902, 1996.

4. Ozols RF, Bundy BN, Greer BE, et al: Phase III trial of carboplatin and paclitaxel compared with cisplatin and paclitaxel in patients with optimally resected stage III ovarian cancer: a Gynecologic Oncology Group study. J Clin Oncol 21: 3194-3200, 2003 
5. Zeimet AG and Marth C: Why did p53 gene therapy fail in ovarian cancer? Lancet Oncol 4: 415-422, 2003.

6. See HT and Kavanagh JJ: Novel agents in epithelial ovarian cancer. Cancer Invest 22: 29-44, 2004.

7. Raki M, Rein DT, Kanerva A and Hemminki A: Gene transfer approaches for gynecological diseases. Mol Ther 14: 154-163, 2006.

8. Rocconi RP, Numnum TM, Stoff-Khalili M, Makhija S, Alvarez RD and Curiel DT: Targeted gene therapy for ovarian cancer. Curr Gene Ther 5: 643-653, 2005.

9. Wolf JK and Jenkins AD: Gene therapy for ovarian cancer (Review). Int J Oncol 21: 461-468, 2002.

10. Buller RE, Runnebaum IB, Karlan BY, et al: A phase I/II trial of $\mathrm{rAd} / \mathrm{p} 53$ (SCH 58500) gene replacement in recurrent ovarian cancer. Cancer Gene Ther 9: 553-566, 2002.

11. Alvarez RD, Barnes MN, Gomez-Navarro J, et al: A cancer gene therapy approach utilizing an anti-erbB-2 single-chain antibody-encoding adenovirus (AD21): a phase I trial. Clin Cancer Res 6: 3081-3087, 2000.

12. Hasenburg A, Tong XW, Fischer DC, et al: Adenovirusmediated thymidine kinase gene therapy in combination with topotecan for patients with recurrent ovarian cancer: 2.5-year follow-up. Gynecol Oncol 83: 549-554, 2001.

13. Alvarez RD and Curiel DT: A phase I study of recombinant adenovirus vector-mediated intraperitoneal delivery of herpes simplex virus thymidine kinase (HSV-TK) gene and intravenous ganciclovir for previously treated ovarian and extraovarian cancer patients. Hum Gene Ther 8: 597-613, 1997.

14. Mahasreshti PJ, Kataram M, Wu H, et al: Ovarian cancer targeted adenoviral-mediated mda-7/IL-24 gene therapy. Gynecol Oncol 100: 521-532, 2006.

15. Bischoff JR, Kirn DH, Williams A, et al: An adenovirus mutant that replicates selectively in p53-deficient human tumor cells. Science 274: 373-376, 1996 .

16. McDermott MF: TNF and TNFR biology in health and disease. Cell Mol Biol 47: 619-635, 2001.

17. Mueller H: Tumor necrosis factor as an antineoplastic agent: pitfalls and promises. Cell Mol Life Sci 54: 1291-1298, 1998

18. Grunhagen DJ, Brunstein F, ten Hagen TL, van Geel AN, de Wilt JH and Eggermont AM: TNF-based isolated limb perfusion: a decade of experience with antivascular therapy in the management of locally advanced extremity soft tissue sarcomas. Cancer Treat Res 120: 65-79, 2004.

19. Weichselbaum RR, Kufe DW, Hellman S, et al: Radiationinduced tumour necrosis factor-alpha expression: clinical application of transcriptional and physical targeting of gene therapy. Lancet Oncol 3: 665-671, 2002.

20. Sharma A, Mani S, Hanna N, et al: Clinical protocol. An openlabel, phase I, dose-escalation study of tumor necrosis factoralpha (TNFerade Biologic) gene transfer with radiation therapy for locally advanced, recurrent, or metastatic solid tumors. Hum Gene Ther 12: 1109-1131, 2001.

21. Senzer N, Mani S, Rosemurgy A, et al: TNFerade biologic, an adenovector with a radiation-inducible promoter, carrying the human tumor necrosis factor alpha gene: a phase I study in patients with solid tumors. J Clin Oncol 22: 592-601, 2004.

22. Wickham TJ: Ligand-directed targeting of genes to the site of disease. Nat Med 9: 135-139, 2003.

23. Bergelson J, Cunningham J, Droguett G, et al: Isolation of a common receptor for Coxsackie B viruses and adenoviruses 2 and 5. Science 275: 1320-1323, 1997.

24. Kirby I, Davison E, Beavil AJ, et al: Mutations in the DG loop of adenovirus type 5 fiber knob protein abolish high-affinity binding to its cellular receptor CAR. J Virol 73: 9508-9514, 1999.

25. Roelvink PW, Mi Lee G, Einfeld DA, Kovesdi I and Wickham TJ: Identification of a conserved receptor-binding site on the fiber proteins of CAR-recognizing adenoviridae. Science 286 : 1568-1571, 1999.

26. Wickham TJ, Mathias P, Cheresh DA and Nemerow GR: Integrins alpha v beta 3 and alpha v beta 5 promote adenovirus internalization but not virus attachment. Cell 73: 309-319, 1993.

27. Wickham TJ, Carrion ME and Kovesdi I: Targeting of adenovirus penton base to new receptors through replacement of its RGD motif with other receptor-specific peptide motifs. Gene Ther 2: 750-756, 1995.

28. Zeimet AG, Muller-Holzner E, Schuler A, et al: Determination of molecules regulating gene delivery using adenoviral vectors in ovarian carcinomas. Gene Ther 9: 1093-1100, 2002.
29. Kelly FJ, Miller CR, Buchsbaum DJ, et al: Selectivity of TAG-72targeted adenovirus gene transfer to primary ovarian carcinoma cells versus autologous mesothelial cells in vitro. Clin Cancer Res 6: 4323-4333, 2000.

30. Lo HW, Day CP and Hung MC: Cancer-specific gene therapy. Adv Genet 54: 235-255, 2005

31. Kufe D, Inghirami G, Abe M, Hayes D, Justi-Wheeler H and Schlom J: Differential reactivity of a novel monoclonal antibody (DF3) with human malignant versus benign breast tumors. Hybridoma 3: 223-232, 1984.

32. Finn OJ, Jerome KR, Henderson RA, et al: MUC-1 epithelial tumor mucin-based immunity and cancer vaccines. Immunol Rev 45: 61-89, 1995.

33. Tai YT, Strobel T, Kufe D and Cannistra SA: In vivo cytotoxicity of ovarian cancer cells through tumor-selective expression of the BAX gene. Cancer Res 59: 2121-2126, 1999.

34. Feng H, Ghazizadeh M, Konishi $\mathrm{H}$ and Araki T: Expression of MUC1 and MUC2 mucin gene products in human ovarian carcinomas. Jpn J Clin Oncol 32: 525-529, 2002.

35. Tsukuda K, Wiewrodt R, Molnar-Kimber K, Jovanovic VP and Amin KM: An E2F-responsive replication-selective adenovirus targeted to the defective cell cycle in cancer cells: potent antitumoral efficacy but no toxicity to normal cell. Cancer Res 62: 3438-3447, 2002.

36. Parr MJ, Manome Y, Tanaka T, et al: Tumor-selective transgene expression in vivo mediated by an E2F-responsive adenoviral vector. Nat Med 3: 1145-1149, 1997.

37. Gu J, Andreeff M, Roth JA and Fang B: hTERT promoter induces tumor-specific Bax gene expression and cell killing in syngenic mouse tumor model and prevents systemic toxicity. Gene Ther 9: 30-37, 2002.

38. Kanerva A, Bauerschmitz GJ, Yamamoto M, et al: A cyclooxygenase-2 promoter-based conditionally replicating adenovirus with enhanced infectivity for treatment of ovarian adenocarcinoma. Gene Ther 11: 552-559, 2004.

39. Kurihara T, Brough DE, Kovesdi I and Kufe DW: Selectivity of a replication-competent adenovirus for human breast carcinoma cells expressing the MUC1 antigen. J Clin Invest 106: 763-771, 2000.

40. Bauerschmitz GJ, Guse K, Kanerva A, et al: Triple-targeted oncolytic adenoviruses featuring the cox 2 promoter, E1A transcomplementation, and serotype chimerism for enhanced selectivity for ovarian cancer cells. Mol Ther 14: 164-174, 2006.

41. Brough DE, Lizonova A, Hsu C, Kulesa VA and Kovesdi I: A gene transfer vector-cell line system for complete functional complementation of adenovirus early regions E1 and E4. J Virol 70: 6497-6501, 1996.

42. Rasmussen H, Rasmussen C, Lempicki M, et al: TNFerade Biologic: preclinical toxicology of a novel adenovector with a radiation-inducible promoter, carrying the human tumor necrosis factor alpha gene. Cancer Gene Ther 9: 951-957, 2002.

43. Einfeld DA, Schroeder R, Roelvink PW, et al: Reducing the native tropism of adenovirus vectors requires removal of both CAR and integrin interactions. J Virol 75: 11284-11291, 2001.

44. Akiyama M, Thorne S, Kirn D, et al: Ablating CAR and integrin binding in adenovirus vectors reduces nontarget organ transduction and permits sustained bloodstream persistence following intraperitoneal administration. Mol Ther 9: 218-230, 2004.

45. Wu S, Boyer CM, Whitaker RS, et al: Tumor necrosis factor alpha as an autocrine and paracrine growth factor for ovarian cancer: monokine induction of tumor cell proliferation and tumor necrosis factor alpha expression. Cancer Res 53: 1939-1944, 1993.

46. Kulbe H, Thompson R, Wilson JL, et al: The inflammatory cytokine tumor necrosis factor-alpha generates an autocrine tumor-promoting network in epithelial ovarian cancer cells. Cancer Res 67: 585-592, 2007.

47. Pikarsky E, Porat RM, Stein I, et al: NF-kappaB functions as a tumour promoter in inflammation-associated cancer. Nature 431: 461-466, 2004.

48. Hemminki A, Belousova N, Zinn KR, et al: An adenovirus with enhanced infectivity mediates molecular chemotherapy of ovarian cancer cells and allows imaging of gene expression. Mol Ther 4: 223-231, 2001.

49. Dmitriev I, Krasnykh V, Miller CR, et al: An adenovirus vector with genetically modified fibers demonstrates expanded tropism via utilization of a coxsackievirus and adenovirus receptorindependent cell entry mechanism. J Virol 72: 9706-9713, 1998. 
50. Liapis H, Adler LM, Wick MR and Rader JS: Expression of alpha(v)beta3 integrin is less frequent in ovarian epithelial tumors of low malignant potential in contrast to ovarian carcinomas. Hum Pathol 28: 443-449, 1997.

51. Witz CA, Montoya-Rodriguez IA, Miller DM, Schneider BG and Schenken RS: Mesothelium expression of integrins in vivo and in vitro. J Soc Gynecol Investig 5: 87-93, 1998.

52. Vanderkwaak TJ, Wang M, Gomez-Navarro J, et al: An advanced generation of adenoviral vectors selectively enhances gene transfer for ovarian cancer gene therapy approaches. Gynecol Oncol 74: 227-234, 1999.

53. Chen L, Cheng DS, Manome Y, Dong YH, Fine HA and Kufe DW: Breast cancer selective gene expression and therapy mediated by recombinant adenoviruses containing the DF3/ MUC1 promoter. J Clin Invest 96: 2775-2782, 1995.
54. Rein DT, Breidenbach M, Kirby TO, et al: A fiber-modified, secretory leukoprotease inhibitor promoter-based conditionally replicating adenovirus for treatment of ovarian cancer. Clin Cancer Res 11: 1327-1335, 2005.

55. Wu H, Han T, Lam JT, et al: Preclinical evaluation of a class of infectivity-enhanced adenoviral vectors in ovarian cancer gene therapy. Gene Ther 11: 874-878, 2004.

56. Wang M, Hemminki A, Siegal GP, et al: Adenoviruses with an RGD-4C modification of the fiber knob elicit a neutralizing antibody response but continue to allow enhanced gene delivery. Gynecol Oncol 96: 341-348, 2005. 